\title{
PRELIMINARY STUDY ON THE NATURAL FREQUENCY OF ADDITIVELY MANUFACTURED BCC LATTICE STRUCTURE WITH HOLLOW STRUTS
}

\author{
MUHAMAD SYAFWAN AZMI ${ }^{1} \&$ RAINAH ISMAIL ${ }^{2}$ \\ ${ }^{I}$ Faculty of Mechanical Engineering, UniversitiTeknikal Malaysia Melaka, Malaysia \\ ${ }^{2}$ Centre for Advanced Research on Energy, UniversitiTeknikal Malaysia Melaka, Malaysia
}

\begin{abstract}
Lattice structure has drawn much attention to researcher due to its vast advantages such as lightweight and high strength to weight ratio. This study presents the preliminary study on the effect of hollow struts of body-centered-cubic (BCC) lattice structure on the natural frequency values vibration characteristic. The samples in this study were made by using fused deposition modeling (FDM) additive manufacturing into cubic and bar samples for compression and vibration test respectively. As expected, through compression test, it was found sample with highest solid strut diameter produced the highest stiffness value followed by hollow strut of the same strut size with 2 mm wall thickness and lastly solid sample with smaller strut diameter size. Meanwhile from vibration test it was found sample with hollow strut produced comparable natural frequency values as sample with highest strut diameter size despite of smaller stiffness.These results suggest that in order to have higher natural frequency values, hollow struts lattice is preferable as it can produce similar natural frequency values with adequately less mass which in the end can reduce cost and material consumption.
\end{abstract}

KEYWORDS: Lattice Structure, 3D Printing, Additive Manufacturing, Natural Frequency \& Compression

Received: Jun 10, 2020; Accepted: Jun 30, 2020; Published: Jul 21, 2020; Paper Id.: IJMPERDJUN2020399

\section{INTRODUCTION}

Lattice structure is one type of cellular structure made up of interconnected network of plates or struts that exist in many forms ranging from natural to human made (Gibson et al., 1999). Lattice structures was initiated from the approximation approach of the foam cellular structures (Hasan, 2013). Lattice structures is periodic structure that are constructed from periodically repeating unit cells and can be considered the improved version of foam structure as it eliminates the uneven mechanical performance of foam structure (Ashby et al., 2000; Ashby, 2006; Cheung et al., 2013). Besides that, lattice structure is also isotropic unlike that of honeycomb's anisotropic behavior as the strength of honeycomb structures is drastically declined when the force exerted is in plane to the honeycomb structures (Cricrì et al., 2013).

Early days of fabrication of the lattice structure was through the conventional manufacturing techniques such as investment casting, hot press method, wire lay-up etc. (Wadley, 2006; Zhang et al., 2012; Lou et al., 2014; Li et al., 2015; Yin et al., 2011). These methods although still work, it can severely restrict the design freedoms of lattice structure due to its principles and limitations. The emergences of additive manufacturing techniques since 1980's pioneered by stereolithography (SLA) technique, enables higher degree of design freedom where the cell size design parameter now can be made into wider range from large to micro scale lattice structures (Hasan, 2013). The exploration on the potential of lattice structures can be done by greater extent as additive manufacturing opens up new opportunity due to its higher degree of design freedom, various materials availability from polymers, metals 
and alloys based and wider range of size availability from macro to micro which is nearly not possible with the use of the conventional manufacturing techniques (Chua et al., 2007; Adam et al., 2014; Ngo et al.,2018).

The exploration on the behavior of lattice structures are usually done through either variation of design parameters or printing process parameters of additively manufactured lattice structure. This is because of the high influence of different design such as topological designs, unit cell's size, variable wall thickness of hollow struts lattices etc. as well as different printing process parameters such as layer thickness, pattern, infill density etc. have different effect on the performance of lattice structure (Azmi et al.,2018). Queheillalt et al., (2005) studied diamond "textile" lattice truss structures with hollow struts and found that to be significantly stronger than their solid truss counterparts. This is because hollow struts have higher second area moments than solid struts. Besides that, Queheillaltet al., (2005) explored the compressive strength of pyramidal lattice structures with hollow struts/trusses made by using the lay-up process conventional manufacturing technique. They found that the compressive strength of a hollow lattice with a relative density of $2.8 \%$ was approximately twice that of a solid pyramidal lattice of similar relative density. A more recent study by Wang et al., (2018) then explored the lattice structure with hollow struts of variable wall thickness manufactured using additive manufacturing and improved the design efficiency by developing a new generative design method where the wall thickness of struts are varied to obtain the optimal materials distribution by means of a mapping method, which avoids complex topology optimization. Therefore, preliminary study was conducted to see the behavior of the lattice structure produced by using fused deposition modeling (FDM) additive manufacturing on natural frequencies values in the dynamic vibration testing as well as compression test.

\section{EXPERIMENTAL PROCEDURES}

\subsection{Sample Fabrication}

Two solid and a hollow struts lattice structure samples based on body centered cubic (BCC) topological design with unit cell size of $160 \times 40 \times 20 \mathrm{~mm}$ in the $8 \times 2 \times 1$ configuration were made for vibration testing by utilizing the CubePro FDM 3D printer. Meanwhile, cubic sample of $20 \times 20 \times 20 \mathrm{~mm}$ in the $2 \times 2 \times 2$ configuration were made compression test. The default process parameters' combination of $200 \mu \mathrm{m}$ layer thickness, solid print strength and diamond print pattern were used for all cubic samples. The sample details as tabulated in Table 1. All samples were made from acrylonitrile butadiene styrene (ABS) material density, $\rho=1.05 \mathrm{~g} / \mathrm{cm}^{\wedge} 3$. Figure 1 shows the illustration of tessellated lattice structure, one-unit cell lattice structure and the outer and inner diameters of the struts.

Table 1: Cubic lattice structure sample details

\begin{tabular}{|c|c|c|c|c|c|}
\hline Sample & $\begin{array}{c}\text { Layer thickness } \\
(\boldsymbol{\mu m})\end{array}$ & Print strength & Print pattern & $\begin{array}{c}\text { Outer diameter } \\
(\mathbf{m m})\end{array}$ & $\begin{array}{c}\text { Inner diameter } \\
(\mathbf{m m})\end{array}$ \\
\hline $4 \mathrm{~mm}$ & 200 & Solid & Diamond & 4 & 0 \\
\hline $5 \mathrm{~mm}$ & 200 & Solid & Diamond & 5 & 0 \\
\hline $5,3 \mathrm{~mm}$ & 200 & Solid & Diamond & 5 & 3 \\
\hline
\end{tabular}




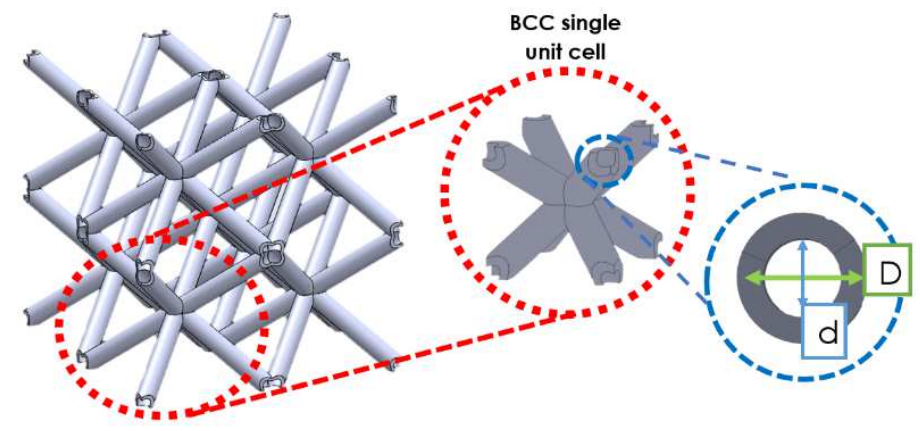

Figure 1: An illustration of tessellated lattice structure, one-unit cell lattice structure and the outer diameter (D) and inner diameter (d) of the struts

\subsection{CompressionTest}

Cubic samples were compressed using universal compression test machine, the Instron 5585 following ASTM D695-15 as guideline. The schematic diagram of compression is shown in Figure 2. The compression test was carried out by applying axial compression at speed rate of $1.5 \mathrm{~mm} / \mathrm{min}$ until densification of the samplesoccurred. The readings acquired from the test were recorded using Blue Hill software. Three compression tests were carried out for sample and the average force and displacement were used in the plot of force against displacement.

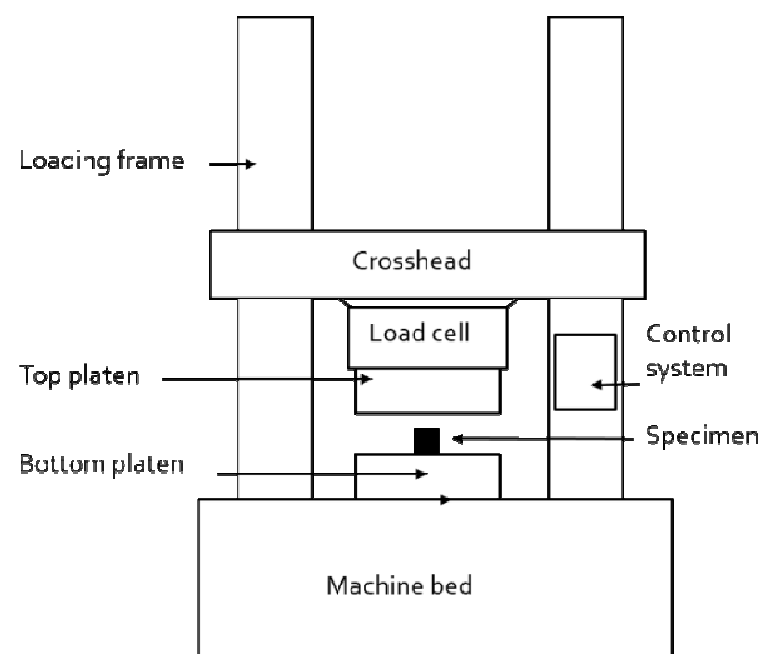

Figure 2: Schematic diagram of the compression machine

\subsection{VibrationTest}

Vibration experiments were carried out to find the natural frequency values of each lattice structure bar. The experimental set-up for vibration measurement consisted of Dataphysics Quattro as signal generator and analyzer, impact hammer and accelerometer sensor to measure the vibration amplitude. Each bar sample was clamped to a solid test rig at one end. A schematic layout of the experimental setup is shown in Figure 3. 


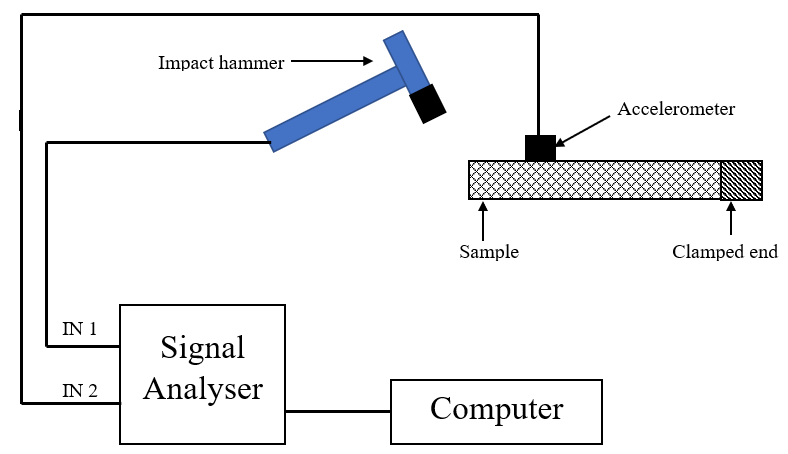

Figure 3: Schematic diagram of the vibration set up

\section{RESULTS AND DISCUSSIONS}

\subsection{CompressionTesting}

The results of the compression tests for each strut diameter were averaged and plotted into force against displacement graph. Figure 4 shows the force-displacement plots of averaged results of compression for each cubic sample. The values of mass and stiffness for each sample then tabulated into Table 2. For the ease of understanding, area moment of inertia of a cross sectional area of single strut is also calculated and tabulated in Table 2 where for sample 5'3, the area moment of inertia is calculated by simply subtracting the moment of inertia of inner diameter from outer diameter.Figure 5 shows the cubic sample undergoes compression.Meanwhile Figure 6 illustrates the cut-up version of Figure 4 which shows the linear part of the graph where the gradients of the linear part provide the stiffness.

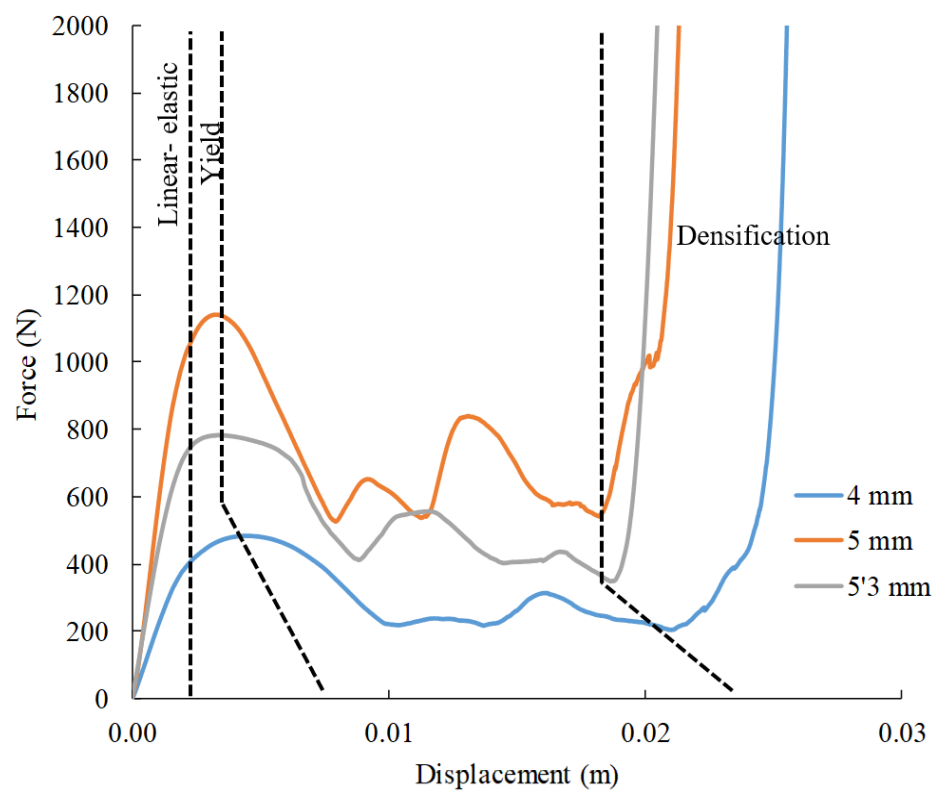

Figure 4: Force-displacement plots for compressed cubic samples 
(a)

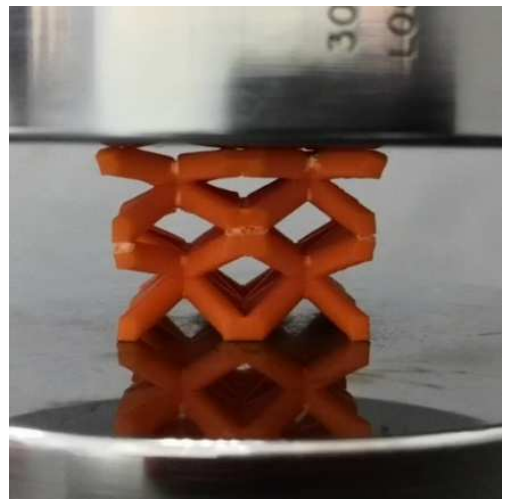

(b)

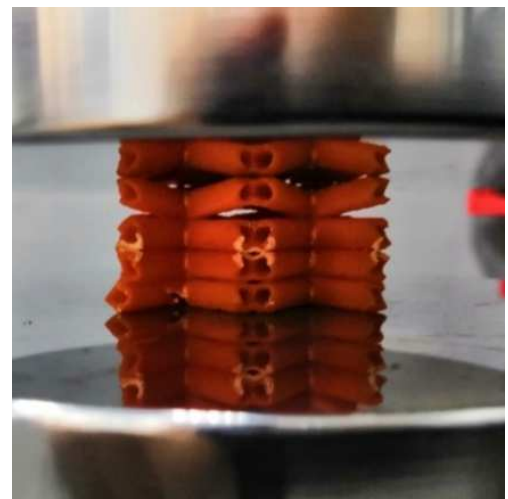

Figure 5: Cubic sample undergoes compression showing (a) sequential struts collapse

(b) complete struts collapse before densification

From Figure 4, it can be seen at low displacement region, linear elastic behavior was shown where the gradients of each plots provide the stiffness. The linear elastic region ended when plastic collapsed. The plots followed by fluctuated curves wherethe lattices destruct in a sequence as thestruts layer collapses, each with its own linear elastic region up to densification regiondue to collapsed cells being forced into contact leading to huge jump in stiffness(Maskery et al., 2017). Based on the cut up linear part in Figure 6, it can be seen that the $5 \mathrm{~mm}$ sample has the highest gradient followed by 5'3 mm sample and lastly $4 \mathrm{~mm}$ sample.
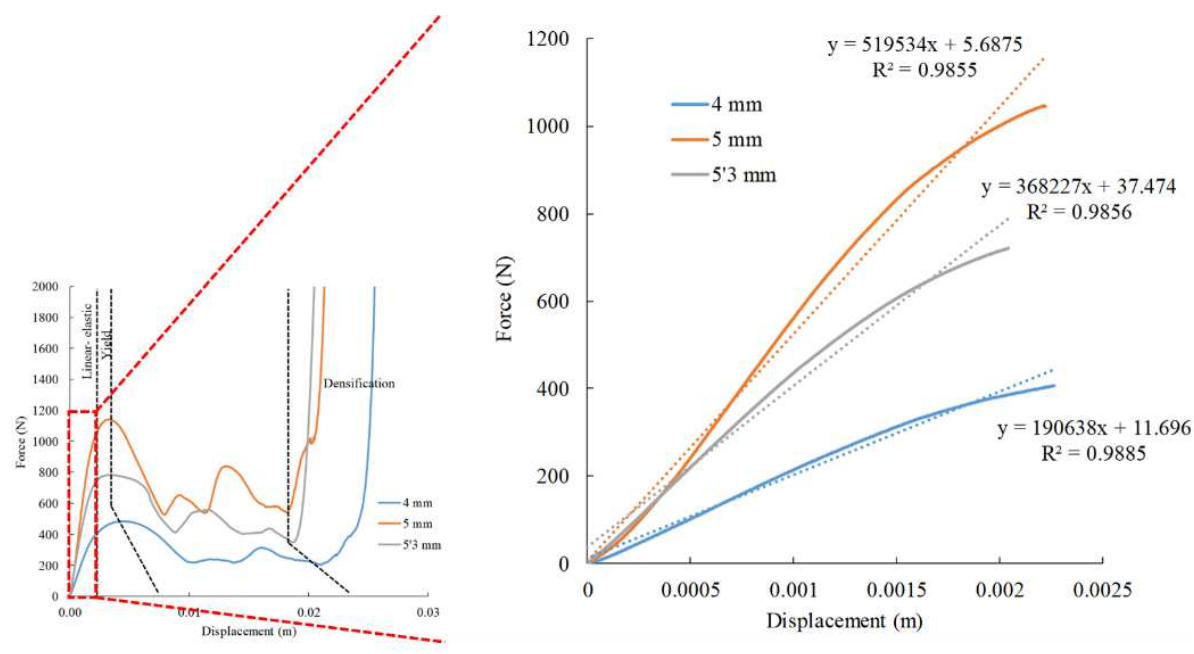

Figure 6: Illustration of cut-up linear-elastic part from force-displacement plots

Table 2: Mass, calculated area moment of inertia and stiffness of samples. Calculated area moment of inertia is calculated forcross sectional area of a single strut

\begin{tabular}{|c|c|c|c|}
\hline Sample & Mass $(\mathbf{g})$ & $\begin{array}{c}\text { Calculated area moment of inertia, } \\
\boldsymbol{I}\left(\mathbf{x ~ e}^{-\mathbf{1 1}} \mathbf{~ m}^{\mathbf{4}}\right)\end{array}$ & Stiffness $(\mathbf{k N} / \mathbf{m})$ \\
\hline $4 \mathrm{~mm}$ & 9.71 & 1.2566 & 190.638 \\
\hline $5 \mathrm{~mm}$ & 14.84 & 3.0680 & 519.534 \\
\hline $5,3 \mathrm{~mm}$ & 11.51 & 2.6704 & 368.227 \\
\hline
\end{tabular}

From Table 2, the values showed that the $5 \mathrm{~mm}$ cube sample exhibit $92.62 \%$ higher percentage different in stiffness when compared to $4 \mathrm{~mm}$ sample and just $34.09 \%$ higher stiffness when compared to sample 5’3 mm.This resultgives insight that sample 5'3 has higher strength to weight ratio as compared to $4 \mathrm{~mm}$ sample as it produced $63.55 \%$ higher percentage different stiffness with only $16.97 \%$ higher percentage different in mass. The trends of stiffness are 
consistent with the calculated area moment of inertia of a cross sectional area of single strut. The trend of stiffness is similar with other study conducted by Syam et al.,(2018) where lattice samples with higher moment of inertia has higher stiffness values.

\subsection{Vibration Testing}

The accelerance FRF and bode plot graphs for sample with $4 \mathrm{~mm}, 5 \mathrm{~mm}$ and 5'3 mm were plotted using MATLABsoftware and shown in Figure 7, Figure 8 and Figure 9 respectively. The first two natural frequency values were also tabulated into Table 3. It can be seen on all three Figure 7, Figure 8 and Figure 9, that the bode-plot has sharp phase shift $90^{\circ}$ angle at resonant frequency.
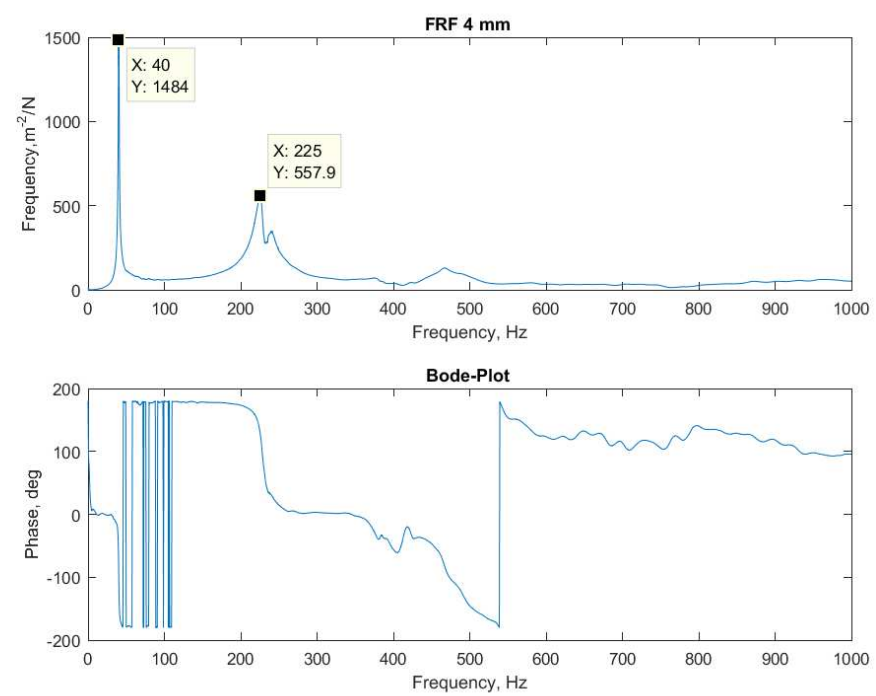

Figure 7: FRF graph and bode plot for sample $4 \mathbf{~ m m}$
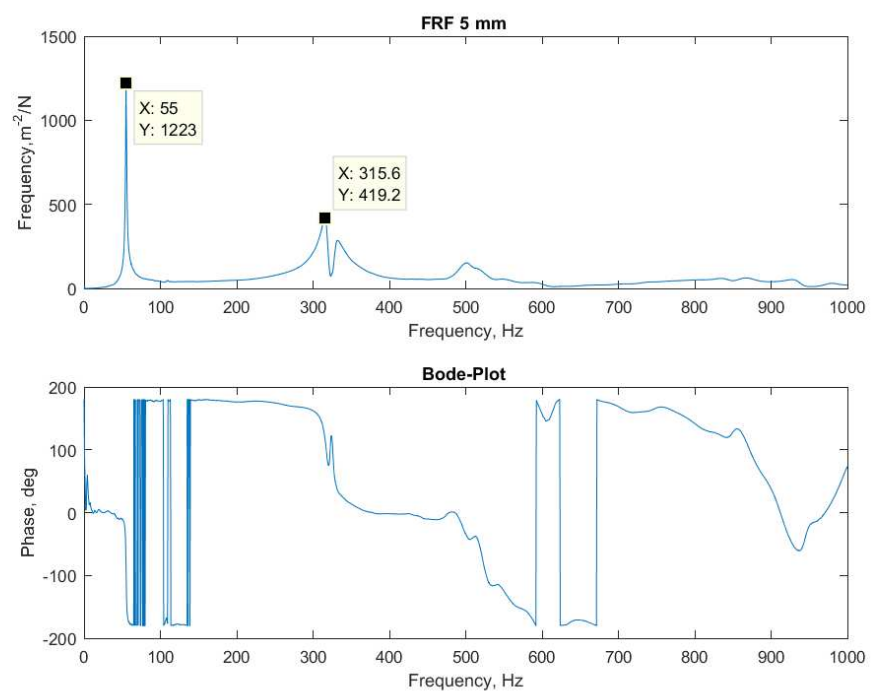

Figure 8: FRF graph and bode plot for sample 5 mm 

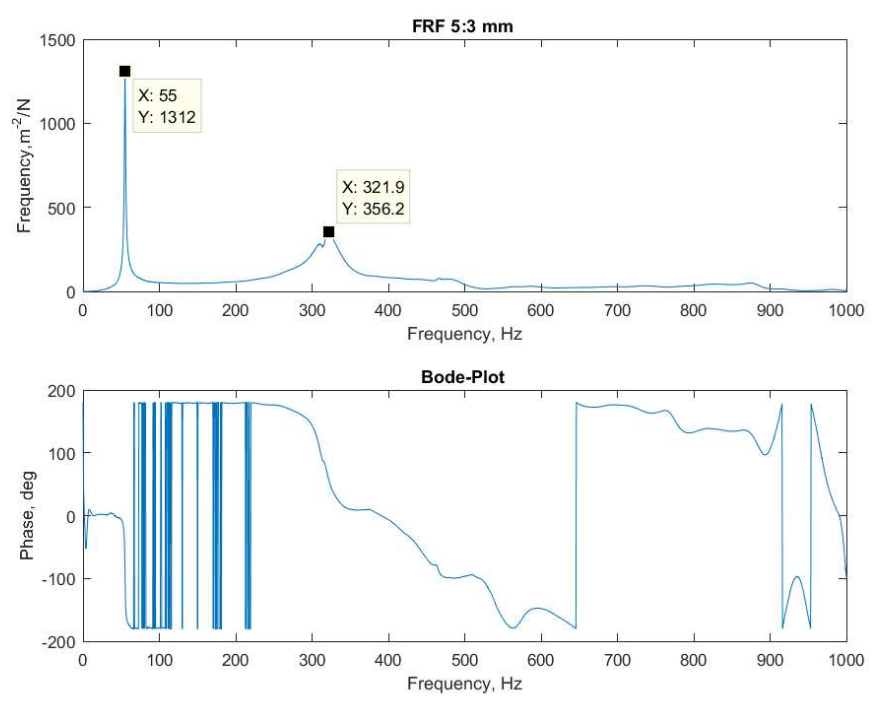

Figure 9: FRF graph and bode plot for sample 5’3 mm

Table 3: Vibration test results and mass of lattice cube samples

\begin{tabular}{|c|c|c|c|}
\hline \multirow{2}{*}{ Sample } & \multirow{2}{*}{ Mass (g) } & \multicolumn{2}{|c|}{ Natural Frequency (Hz) } \\
\cline { 3 - 4 } & & 1st Mode & 2nd Mode \\
\hline $4 \mathrm{~mm}$ & 19.566 & 40.0 & 225.0 \\
\hline $5 \mathrm{~mm}$ & 29.799 & 55.0 & 315.6 \\
\hline $5,3 \mathrm{~mm}$ & 23.115 & 55.0 & 321.0 \\
\hline
\end{tabular}

Based on Table 3, it can be seen that the $5 \mathrm{~mm}$ sample exhibited similar first mode natural frequency values with sample of 5'3 mm struts and slightly lower second mode natural frequency to that of $5 \mathrm{~mm}$ sample. Meanwhile, as expected sample with $4 \mathrm{~mm}$ strut exhibited the lowest both first and second modes natural frequency values. Equation (1) shows the formula of natural frequency where $\omega_{n}$ is the natural frequency $(\mathrm{Hz}), k$ is the stiffness $(\mathrm{N})$ and $m$ is mass $(\mathrm{kg})$.

$$
\omega_{n}=\sqrt{\frac{k}{m}}
$$

Based on equation (1), the mass of the lattice structure cellular material bar samples is supposedly inversely proportional to the natural frequency (i.e. an increase in mass will lower the natural frequency). However, the from sample $5 ’ 3 \mathrm{~mm}$ and $4 \mathrm{~mm}$ showed otherwise. Thus, this suggests that despite an increase in mass of the lattice structure from sample 5'3 mm and $4 \mathrm{~mm}$, the stiffness component of the lattice structure is more significant compared to the mass component of the lattice structure cellular material bar samples. Nonetheless, despite having higher stiffness for sample 5 $\mathrm{mm}$, the natural frequency values are comparable to that of $5,3 \mathrm{~mm}$ as it is lighter. These results suggest that in order to have higher natural frequency values, hollow struts lattice is preferable as it can produce similar natural frequency values with adequately less mass which in the end can reduce cost and material consumption.

\section{CONCLUSIONS}

Through the hollow strut design parameter with ( $2 \mathrm{~mm}$ wall thickness), comparable natural frequency to that of solid strut counterpart can be achieved. This shows that the variable wall thickness design parameter has significant influence to the natural frequency values. Although from the compression test, the hollow sample 5'3produced less stiffness. However, due to smaller mass value, similar natural frequency can be achieved. The results suggest that in order to have higher natural 
frequency values, hollow struts lattice is preferable as it can reduce cost as well as material consumption for sustainability.

\section{ACKNOWLEDGEMENT}

The authors acknowledge the financial support from Zamalah Scheme, UniversitiTeknikal Malaysia Melaka.

\section{REFERENCES}

1. Adam, G. A., \& Zimmer, D. (2014). Design for Additive Manufacturing-Element transitions and aggregated structures. CIRP Journal of Manufacturing Science and Technology, 7(1), 20-28.

2. Ashby, M. F. (2006). The properties of foams and lattices. Philosophical Transactions of the Royal Society A: Mathematical, Physical and Engineering Sciences, 364(1838), 15-30.

3. Ashby, M. F., Evans, T., Fleck, N. A., Hutchinson, J. W., Wadley, H. N. G., \& Gibson, L. J. (2000). Metal foams: a design guide. Elsevier.

4. Alizadeh, Asad, And Anas Abid Mattie. "Two-Dimensional Simulation To Investigate The Interaction Of Fluid-Structure Inside A Microchannel With Elastic And Rigid Boundary."International Journal of Mechanical and Production Engineering Research and Development (IJMPERD) 9. 4, Aug 2019, 1151-1156

5. Azmi, M. S., Hasan, R., Ismail, R., Rosli, N. A., \& Alkahari, M. R. (2018). Static and dynamic analysis of FDM printed lattice structures for sustainable lightweight material application. Progress in Industrial Ecology, an International Journal, 12(3), $247-259$.

6. Cheung, K. C., \& Gershenfeld, N. (2013). Reversibly assembled cellular composite materials. science, 341(6151), 1219-1221.

7. Chua, C. K., Leong, K. F., \& Lim, C. S. (2010). Rapid prototyping: principles and applications (with companion CD-ROM). World Scientific Publishing Company.

8. Peddi, Siva Prasad, And Bilal Abdallah Sadeh. "Study Of The Contraction Of The Lattice Parameters Of Silver Nanoparticles Synthesized Using Achyranthes Aspera L Stem Extract."International Journal of Nanotechnology and Application (IJNA) 5.3, Jun 2015, 1-12

9. Cricrì, G., Perrella, M., \& Calì, C. (2013). Honeycomb failure processes under in-plane loading. Composites Part B: Engineering, 45(1), 1079-1090.

10. Gibson, L. J., \& Ashby, M. F. (1999). Cellular solids: structure and properties. Cambridge university press.

11. Hasan, R. (2013). Progressive collapse of titanium alloy micro-lattice structures manufactured using selective laser melting (Doctoral dissertation, University of Liverpool).

12. Xydou, A., et al. "Production of CLIC accelerating structures using diffusion bonding at very high temperature." Int. Journal of Mettalurgical and Materials Science and Engineering (IJMMSE) 6.2 (2016).

13. Li, B., Li, Z., Zhou, J., Ye, L., \& Li, E. (2015). Damage localization in composite lattice truss core sandwich structures based on vibration characteristics. Composite Structures, 126, 34-51.

14. Lou, J., Wu, L., Ma, L., Xiong, J., \& Wang, B. (2014). Effects of local damage on vibration characteristics of composite pyramidal truss core sandwich structure. Composites part b: Engineering, 62, 73-87.

15. Kumar, T. Vijaya, and K. V. Ramana. "Effect of Ball Milling on microstructural parameters of nanostructured MoO3-CuOV2O5 composite Nano powders." International Journal of Mechanical and Production Engineering Research and Development 9.3 (2019): 131-142. 
16. Maskery, I., Hussey, A., Panesar, A., Aremu, A., Tuck, C., Ashcroft, I., \& Hague, R. (2017). An investigation into reinforced and functionally graded lattice structures. Journal of Cellular Plastics, 53(2), 151-165.

17. Ngo, T. D., Kashani, A., Imbalzano, G., Nguyen, K. T., \& Hui, D. (2018). Additive manufacturing (3D printing): A review of materials, methods, applications and challenges. Composites Part B: Engineering, 143, 172-196.

18. Queheillalt, D. T., \& Wadley, H. N. (2005). Cellular metal lattices with hollow trusses. Acta Materialia, 53(2), 303-313.

19. Syam, W. P., Jianwei, W., Zhao, B., Maskery, I., Elmadih, W., \& Leach, R. (2018). Design and analysis of strut-based lattice structures for vibration isolation. Precision Engineering, 52, 494-506.

20. Wadley, H. N. (2006). Multifunctional periodic cellular metals. Philosophical Transactions of the Royal Society A: Mathematical, Physical and Engineering Sciences, 364(1838), 31-68.

21. Wang, Y., Jing, S., Liu, Y., Song, G., Qie, L., \& Xing, H. (2018). Generative design method for lattice structure with hollow struts of variable wall thickness. Advances in Mechanical Engineering, 10(3), 1687814017752482.

22. Yin, S., Wu, L., Ma, L., \& Nutt, S. (2011). Pyramidal lattice sandwich structures with hollow composite trusses. Composite structures, 93(12), 3104-3111.

23. Zhang, G., Ma, L., Wang, B., \& Wu, L. (2012). Mechanical behaviour of CFRP sandwich structures with tetrahedral lattice truss cores. Composites Part B: Engineering, 43(2), 471-476. 

